\section{The 200-inch Telescope}

According to Science Service, of Washington, D.C., it is now definite that the two hundred-inch telescope will be erected on Palomar Mountain, 45 miles north-east of San Diego, California. Astronomers have never felt completely satisfied with the 'seeing' at Mount Wilson. Bad 'seeing' arises from local irregularities in the atmospheric refraction which have the effect of distorting the stellar image, especially when a very large aperture telescope is being used; and since it was supposed that convection currents of air running up the steep sides of Mount Wilson contributed materially to the imperfections in the seeing at that observatory, a search for better seeing has been conducted on many plateaux and flat-topped peaks in southern California. The tests of seeing were made by observing Polaris through a standard telescope and noting the character of the image from night to night. The flat-topped Palomar has excellent seeing qualities; it is further advantageous in that it is remote from any great city. Scattered light from Los Angeles and its environs finds its way into the 100 -inch reflector on Mount Wilson, and imposes a limit on the length of exposure which one can give to a photographic plate. It may be added that the 200 -inch mirror is to be cast at Corning, N.Y., the scene of a previous 200 -inch cast, now kept as a 'spare', while the mirror will be ground and the telescope itself constructed in Pasadena, at the California Institute of Technology. This institution will be responsible for the administration of the telescope when completed.

\section{A Portable Sundial}

WE have received a small portable sundial of ingenious construction, made of aluminium ("KosmosSonnenuhr". Stuttgart: Franckh'sche Verlagshandlung). The workmanship is neat and attractive, and the instrument should be of value in giving instruction to schoolchildren. Some features call for comment. The sundial can be adapted to work in any latitude by rotating one of its members about an axis, the latitude being read off by a pointer against a scale marked from $0^{\circ}$ to $90^{\circ}$ by steps of $5^{\circ}$. This device, of course, helps the manufacturer to bring out sundials for various latitudes by mass production, and it may conceivably assist a teacher demonstrating fundamental principles to students; but we cannot imagine any real use for a sundial which is to be carried from one latitude to another. A further comment should be made on the method of adjustment in azimuth. A small magnetic compass is embodied in the instrument, and values of the magnetic variation for a number of places are given in the instructions. But if the instrument is to be set up permanently, one had better look up the equation of time in an almanac and set the instrument by adjusting it to give the right time, known independently. It will then, of course, work correctly; and this method of adjustment is easier to effect as well as being more accurate than the use of a tiny compass, liable to gross error through stray magnetic field.

\section{Leaflets of the Astronomical Society of the Pacific}

For some nine years past, the Astronomical Society of the Pacific (Merchants' Exchange Building, San Francisco, California, U.S.A.) has issued-at first irregularly, then bi-monthly, and later, in response to growing demand, once every month-a series of leaflets explaining, in simple but authoritative terms, astronomical matters of general interest. These leaflets have become widely known and appreciated, not only by the general astronomically-minded public for whom they were primarily designed and whose interests have been given first consideration through. out, but also by working astronomers as a convenient and trustworthy source of information on certain facts to which reference is frequently required. For example, in Leaflet 30, Dr. F. C. Leonard, of the University of California, gives an account of the newly-discovered planet, Pluto, with the most accurate data available at the time and a useful diagram showing the relation of the orbit to the orbits of the other planets of the solar system; while the distance-velocity relation characterising the extra-galactic nebulæ is explained with a table, diagram and photograph in Leaflet 37 by $\mathrm{Mr}$. Humason himself. The first fifty of these leaflets, each bearing the date of writing, have now been bound together and re-issued as a small volume, and it is satisfactory to note that, since the monthly publication of the leaflets is to be continued, further volumes may be expected in due time. The collection, which it is impossible to praise too highly, unfortunately bears no indication of its cost, but we feel confident in saying that no one interested in astronomy, whatever his degree of knowledge of the subject, will regret the purchase of a copy.

\section{White and Coloured Headlight Beams in Fog}

Experrments, made at the National Physical Laboratory, comparing the revealing power of white and coloured headlight beams in fog, are described by Dr. W. S. Stiles in the Illuminating Engineer of October. Two headlights of known candle-power distribution were mounted at the height and spacing employed on the average car. They were arranged to throw their beams parallel to and in the direction of the road. The observer stood behind the offside headlight and viewed a disc painted grey with its centre lying on the axis of the light. Test discs of different reflecting powers were used in the experiments. The discs were moved up and down the road, and the distances at which the dises first became invisible were measured. The experiments were repeated with both coloured and neutral light filters held in a frame at a suitable height. Taken as a whole, the results point definitely to the conclusion that the sole effect of the colour filters is due to the reduction in light intensity. This was proved by showing that the range of observations for neutral and colour filters, when plotted against filter transmission, lay on the same curve. The effects that occurred always showed a reduction in the range when compared with the unmodified beam. So far as the experiments went, the revealing power of a 